\title{
Spectroscopy of Nonspherical Atomic Bubbles in Solid Helium
}

\author{
V. Lebedev, ${ }^{*}$ P. Moroshkin, and A. Weis \\ Département de Physique, Université de Fribourg, Chemin du Musée 3, 1700 Fribourg, Switzerland
}

\begin{abstract}
We analyze the effect of the host crystal symmetry on the optical spectra of the $6 P_{1 / 2}-6 S_{1 / 2}$ and $6 P_{3 / 2}-6 S_{1 / 2}$ transitions of atomic Cs in solid ${ }^{4} \mathrm{He}$ matrices. In particular, we address the deformation of the bubble structures formed by Cs in such quantum crystals. We show that the anisotropy of the stiffness tensor leads to static quadrupolar bubble shape deformations in hexagonally close-packed (hcp) crystals, while the corresponding deformations in the body-centered cubic (bcc)
\end{abstract} phase of the matrix have a hexadecupolar symmetry. A compar-
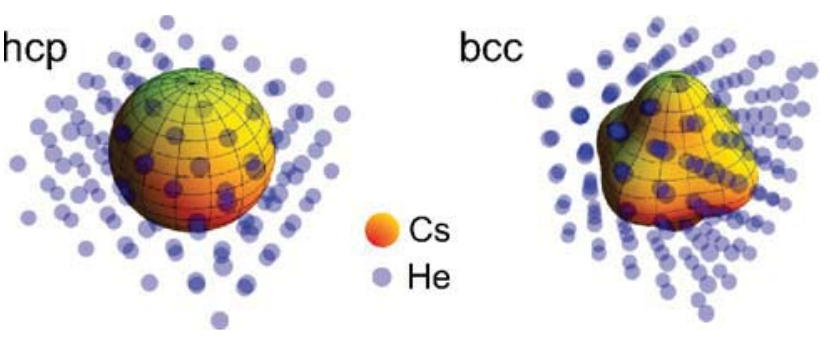
ison of the measured excitation spectra with our model calculations allow us to infer quantitative values of the deformation parameters.

\section{INTRODUCTION}

Matrix isolation spectroscopy is a powerful method for studying chemically reactive, unstable, and weakly bound molecules and complexes. Condensed ${ }^{4} \mathrm{He}$ is the gentlest of all cryogenic matrices in the sense that it perturbs the properties of the isolated species the least, thus, enabling high resolution spectroscopic studies. ${ }^{1,2}$ The low temperature of the host matrix, its chemical inertness, its quantum nature, and diamagnetic character play key roles in this respect. Alkali atoms are model objects for high resolution laser spectroscopy because their electronic structure is relatively simple and can be calculated with a high accuracy. Moreover, alkali atoms possess strong absorption lines in the visible and near-infrared parts of the spectrum. In the present work we have used laser spectroscopy of Cs atoms embedded in solid ${ }^{4} \mathrm{He}$ to characterize the interaction between the dopants and the matrix and to investigate the role played by the symmetry of the host crystal structure.

In solid matrices of the heavier noble gases, the dopant species' properties are strongly perturbed by the local anisotropy of the trapping environment. ${ }^{3}$ Due to its quantum nature, solid ${ }^{4} \mathrm{He}$ is much softer, and the dopant can largely impose its own symmetry on the local trapping site. This is evidenced by the fact that symmetry-dependent alterations of the dopant's properties (for a review see refs 4 and 5), such as distortions and splittings of their optical spectral lines, or the appearance of zero-field magnetic resonance lines, are much less pronounced in helium matrices than in the conventional heavier noble gas matrices. A quantitative understanding of the impurity-matrix interactions and, in particular, the influence of the local trapping site symmetry, is crucial for the interpretation of matrix isolation experiments. Once the matrix-induced modifications of the dopant properties are known, the dopants themselves can be used as probes to characterize the perturbing medium itself on microscopic and nanoscopic scales.

Because of the pronounced quantum delocalization of its constituents, the He matrix can be modeled as a continuous medium, and its interaction with the dopant species can be described using a hydrodynamic model. ${ }^{6}$ The comparatively small anisotropy and large elasticity ${ }^{7,8}$ of solid helium permits treating the effects of local matrix anisotropies on the impurities as small perturbations.

In this paper we will focus on modifications of the optical spectra of embedded Cs atoms induced by the specific anisotropies of body-centered cubic (bcc) and hexagonal close-packed (hcp) crystalline ${ }^{4} \mathrm{He}$ matrices (Figure 1). The main objective is to trace back both the abrupt change of the Cs D-lines' spectral features at the bcc-hcp phase transition and of the $\mathrm{D}_{2}$ absorption line splitting to the atomic bubble deformations induced by the local matrix anisotropy. The goal of this study was to infer bubble deformation parameters from fitting model calculations to experimental data.

The paper is organized as follows. First we introduce the spectra of alkali atoms in condensed helium and review earlier work on atomic bubble deformations. We then present our experimental results on the spectroscopy of Cs atoms in solid helium together with earlier results obtained in liquid helium. A theoretical treatment of static bubble deformations is presented, the results of which are used to infer the deformation parameters from experimental data. Finally, the results are compared with those of earlier treatments. A first appendix reviews the main features of the (previously published) spherical bubble model, and a second appendix presents a generalization of Young's modulus for the specific structures in solid ${ }^{4} \mathrm{He}$, namely, bcc and hcp crystals.

\section{SPECTRA OF SPHERICAL ATOMIC BUBBLES}

Atomic impurities in condensed ${ }^{4} \mathrm{He}$ form nanoscopic cavities, ${ }^{9}$ which are called atomic bubbles, in analogy to electron 


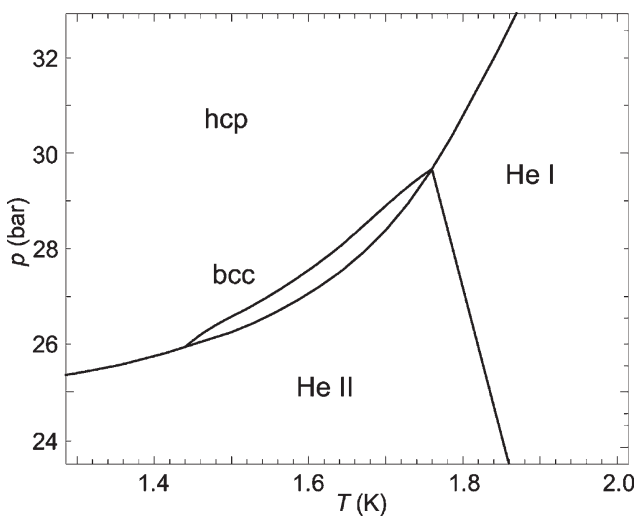

Figure 1. Region of the phase diagram of ${ }^{4} \mathrm{He}$ relevant for the matrix isolation experiments discussed herein.

bubbles formed by isolated electrons in condensed ${ }^{4} \mathrm{He} .{ }^{6}$ This bubble formation is a consequence of the strongly repulsive interaction between the closed electronic shell of the He atoms and the single valence electron of the dopant atom. The shape and size of these cavities are determined first by the shape and size of the dopant's electronic wave function and, second, by the density and elasticity of the helium environment. In its simplest form, this bubble model assumes the He density distribution to be homogeneous, which is justified by the delocalized quantum nature of the $\mathrm{He}$ atoms. It also assumes that the pressure exerted by the matrix on the bubble interface is isotropic, an assumption that is strictly valid only for static interactions in superfluid matrices. As a consequence, electrons, or atoms and molecules in $J=0$ or $J=1 / 2$ states with spherically symmetric wave functions, will form spherically symmetric bubbles.

For a number of years we have studied extensively, both experimentally and theoretically, the first resonance doublet of Cs in solid He. ${ }^{10,11}$ As a main feature, we found that the spectral lines of the $6 S_{1 / 2}-6 P_{1 / 2}\left(D_{1}\right)$ and $6 S_{1 / 2}-6 P_{3 / 2}\left(D_{2}\right)$ transitions are strongly blueshifted and broadened in comparison with the free atomic lines, the blueshift and broadening being more pronounced in the excitation (absorption) spectra than in the fluorescence (emission) spectra. Fluorescence on the Cs $\mathrm{D}_{2}$ transition cannot be observed because of a predominant deexcitation channel toward the formation of excited state quasimolecules (exciplexes). ${ }^{12}$

These features can be qualitatively understood by the excitation-emission cycles that Cs atoms in bubbles undergo by resonant optical excitation (Figure 2). The sizes and shapes of the equilibrium bubbles depend on the electronic states of the dopant atom. The ground state atom is excited while in a small bubble, which strongly perturbs (shifts) the atomic energy levels. The bubble containing the excited state relaxes in a few picoseconds to a larger equilibrium size. The subsequent emission thus occurs from atoms housed in a larger bubble, which itself induces smaller shifts of the energy levels and, hence, of the emitted fluorescence spectrum. Finally, the bubble shrinks back to its original small size, again on a picosecond time scale.

Another property of the alkali lines in helium matrices is the pronounced dependence of their wavelengths on the pressure of the host medium. At fixed temperature, the density increases monotonically with pressure and the line position shows a linear density dependence with different slopes in liquid, bcc solid, and hcp solid helium (Figures 5 and 7).

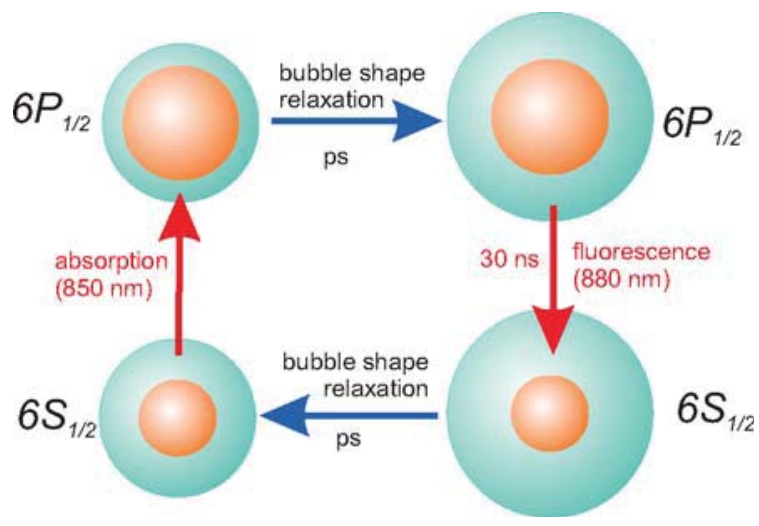

Figure 2. Optical excitation-emission cycle for a Cs atom in solid hcp- ${ }^{4} \mathrm{He}$.

The pressure dependences of the $\mathrm{D}_{1}$ and $\mathrm{D}_{2}$ line centers in excitation and the $\mathrm{D}_{1}$ line in emission show a pronounced step at the liquid-bcc phase transition that can be completely accounted for by the corresponding step in the pressure dependence of the density. Similar steps appear in the pressure dependence of both line centers at the phase transition from bcc to hcp and cannot be explained by the small density difference between the two phases. ${ }^{11,13}$

\section{EVIDENCE FOR ATOMIC BUBBLE DEFORMATIONS}

In solid helium, the bubble shapes formed by spherically symmetric atoms are expected to be deformed because of the anisotropic elasticity of the host matrix. In the past, a number of specific spectroscopic features have been observed that can be associated with such static and dynamic bubble deformations.

The most pronounced effects of deformed bubbles were seen in optically detected magnetic resonance (ODMR) experiments on $\mathrm{Cs}$ atoms in solid ${ }^{4} \mathrm{He}$ (reviewed in refs 4 and 5). The magnetic resonance spectrum of (intramultiplet) transitions between Zeeman sublevels of alkali ground states recorded in $\mu \mathrm{T}$ magnetic fields consists of a single $20 \mathrm{~Hz}$ wide line in bcc, ${ }^{14}$ while in hcp (at similar pressure and temperature), the spectrum broadens to several $\mathrm{kHz}$ and shows a rich substructure. ${ }^{4}$ Hyperfine (intermultiplet) transitions in the $6 S_{1 / 2}$ state were also found to differ for bcc and hcp host matrices, ${ }^{15,16}$ showing a pressuredependent $\approx 2 \%$ blue shift of the clock transition frequency in bcc, and a slightly smaller shift in hcp. Furthermore, magnetic dipole-forbidden $\Delta M=2,3$ transitions were detected in the ODMR spectra in hcp, ${ }^{17}$ while being absent in bcc. Last but not least, we observed ${ }^{16}$ in hcp a magnetic resonance spectrum when no magnetic field was applied (zero-field spectrum). All of these observations have been explained, at least qualitatively, by assuming a small static quadrupolar deformation of the atomic bubble in hcp.

The atomic bubble deformation also manifests itself in the optical absorption and emission spectra of Cs in solid helium. It was suggested ${ }^{11,13}$ that the discontinuity of the pressure dependence of the absorption and fluorescence spectra at the hcp-bcc phase boundary is due to different bubble deformations in the two crystalline phases. It was reported ${ }^{11,18}$ that the $\mathrm{D}_{2}$ absorption spectrum in hcp possesses a pronounced doublet structure (Figure 3) that can be qualitatively explained by a quadrupolar bubble deformation. However, so far, a quantitative description 


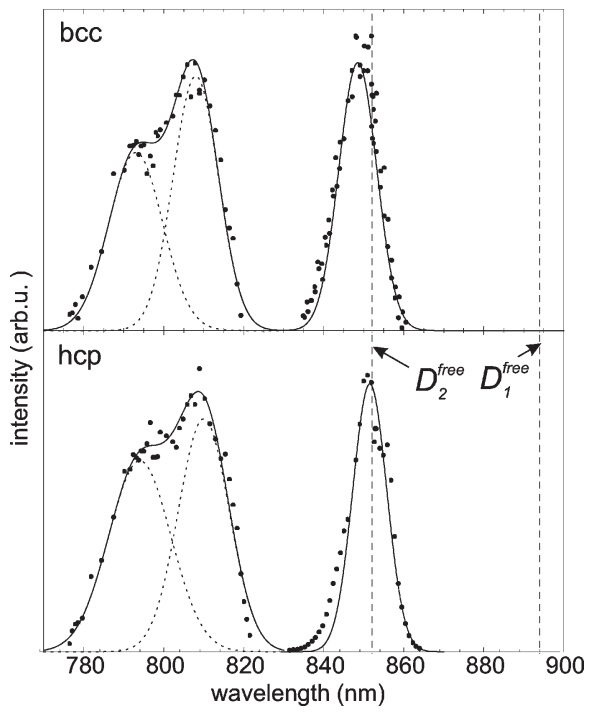

Figure 3. Excitation spectrum of atomic Cs in bcc solid helium (26.5 bar, $1.5 \mathrm{~K}$, top) and in hcp solid helium (27.0 bar, $1.5 \mathrm{~K}$, bottom). Experimental data (dots) are fitted by Gaussians (solid lines). The dashed vertical lines indicate the positions of the $\mathrm{D}_{1}$ and $\mathrm{D}_{2}$ lines in the free Cs atom.

and understanding of corresponding phenomena in optical (electric dipole) transitions has not been addressed.

Descriptions of experimental effects due to dynamic bubble deformations are also scarce. The pronounced doublet splitting of the $\mathrm{Cs}_{2}$ excitation line, also observed in superfluid helium, ${ }^{19}$ was explained in terms of a dynamic Jahn-Teller splitting of the $6 P_{3 / 2}$ state due to quadrupolar bubble oscillations. ${ }^{20}$ Bubbleshape oscillations were also used to explain the observed nonsymmetric line shapes of alkali-earth atoms $(\mathrm{Ca}, \mathrm{Mg})$ and the splitting of the $\mathrm{D}_{2}$ line of $\mathrm{Yb}^{+}$ions in superfluid helium. ${ }^{21-23}$ The long, albeit finite, spin depolarization time observed in ODMR experiments with polarized $\mathrm{Cs}$ atoms in superfluid ${ }^{24,25}$ and bccsolid ${ }^{4} \mathrm{He}^{26}$ has been tentatively explained in terms of a coupling to dynamic quadrupolar bubble oscillations.

\section{EXPERIMENTAL SECTION}

We recorded excitation spectra of the $6 S_{1 / 2}-6 P_{1 / 2}\left(D_{1}\right)$ and $6 S_{1 / 2}-6 P_{3 / 2}\left(D_{2}\right)$ transitions of ${ }^{133} \mathrm{Cs}$ in condensed ${ }^{4} \mathrm{He}$. From these spectra we inferred the line shape parameters (line centers and widths) as a function of helium pressure (density) in superfluid, bcc, and hcp host matrices.

4.1. Experimental Setup. All measurements were performed with a setup that was described in detail in previous publications. ${ }^{4,5,27}$ The Cs-doped solid He sample was prepared in the following way. A helium crystal was grown by pressurizing liquid $\mathrm{He}$ inside of a pressure cell immersed in a superfluid helium bath whose temperature was controlled by pumping on the He surface. The temperature and pressure of the samples can be varied in the range of $1.4-4.2 \mathrm{~K}$ and $1-40$ bar, respectively. The pressure cell has five fused silica windows in orthogonal directions. We used a pulsed laser ablation technique to dope the He sample with Cs atoms. ${ }^{4,5,27}$ Typical atomic Cs densities of $\approx 10^{10} \mathrm{~cm}^{-3}$ were inferred from the intensity of the $\mathrm{D}_{1}$ resonance fluorescence.

Optical transitions of Cs atoms in the sample were excited by the idler beam of an optical parametric oscillator (OPO) pumped

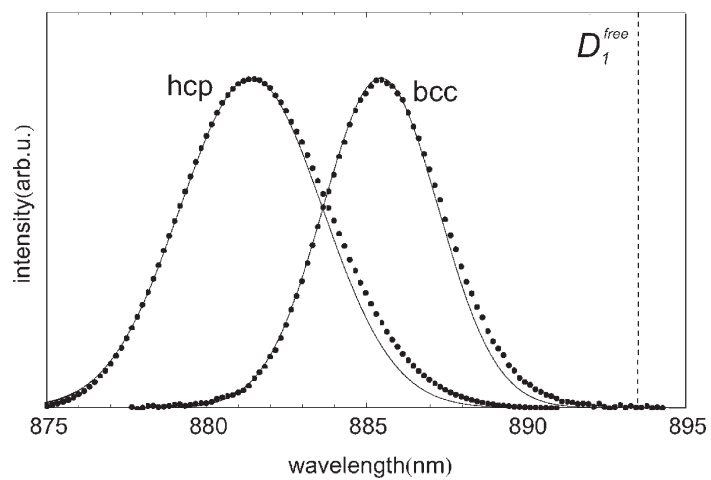

Figure 4. Emission spectra of Cs atoms in bcc solid helium ( $p=26.5$ bar, $T=1.5 \mathrm{~K})$ and in hcp solid helium $(p=27.0 \mathrm{bar}, T=1.5 \mathrm{~K})$. Dots represent the experimental data, Gaussian fits are shown as solid lines. The dashed vertical line denotes the $\mathrm{D}_{1}$ wavelength of the free atom.

by the third harmonic of a Nd:YAG laser. Fluorescence was collected at $90^{\circ}$ and analyzed by a grating spectrograph equipped with a CCD camera (resolution of $\approx 0.12 \mathrm{~nm}=1.5 \mathrm{~cm}^{-1}$ ). Excitation spectra of the $\mathrm{D}_{1}$ and $\mathrm{D}_{2}$ transitions were recorded by tuning the OPO wavelength and measuring (in both cases) the $\mathrm{D}_{1}$ fluorescence intensity. The spectral resolution was largely determined by the spectral width of the exciting OPO radiation and was up to $5.5 \mathrm{~nm}\left(85 \mathrm{~cm}^{-1}\right)$ at the $\mathrm{D}_{2}$ line and $\approx 2 \mathrm{~nm}$ $\left(25 \mathrm{~cm}^{-1}\right)$ at the $\mathrm{D}_{1}$ line.

Typical spectra of excitation and emission lines of Cs atoms are shown in Figure 3. We applied a deconvolution technique to extract the center and width of each spectral line assuming Gaussian line shapes.

4.2. Fluorescence Spectra. Irrespective of the excitation $\left(D_{1}\right.$ or $\left.\mathrm{D}_{2}\right)$, only $\mathrm{D}_{1}$ fluorescence is emitted from the $6 P$ states, because $\mathrm{D}_{2}$ fluorescence (from the $6 P_{3 / 2}$ state) is strongly suppressed by the formation of $\mathrm{Cs}^{*} \mathrm{He}_{n}$ exciplexes and fine structure relaxation toward the $6 P_{1 / 2}$ state. ${ }^{11,12}$ In agreement with earlier work ${ }^{19}$ in superfluid helium, the $\mathrm{D}_{1}$ fluorescence line is considerably blue-shifted with respect to the free atomic line, and the shift increases linearly with helium pressure (density). When crossing the superfluid-bcc phase transition, the line center shows a discontinuous jump of $2 \mathrm{~nm}\left(25 \mathrm{~cm}^{-1}\right)$.

When going from bcc to hcp, a further jump of $2 \mathrm{~nm}\left(25 \mathrm{~cm}^{-1}\right)$ to the blue occurs (Figure 4).

4.3. Excitation Spectra. As shown in Figure 3 , the $D_{2}$ absorption line has a doublet structure with a splitting of $\approx 15 \mathrm{~nm}\left(\approx 200 \mathrm{~cm}^{-1}\right)$. Both lines show an average blue-shift of $40-50 \mathrm{~nm}\left(600-700 \mathrm{~cm}^{-1}\right)$ with respect to the free atom. The centers of all three $\left(D_{2}\right.$ doublet and $\left.D_{1}\right)$ features shift monotonically with $\mathrm{He}$ pressure (density) at different rates in the liquid and solid phases of the He matrix (Figure 5). We have shown earlier ${ }^{13}$ that the discontinuity of the $\mathrm{D}_{1}$ line position at the solidification point can be fully accounted for by the density change at the phase transition, and that it disappears when the line parameters are shown as a function of He density rather than He pressure (Figures 5-7). However, the jump at the bcc-hcp transition persists even in the density-dependence plot. This jump is the center of interest of this paper. Note that the nonaccessible regions between the superfluid (HeII) and bcc phase in Figures 5-7 are due to the abrupt density change at the transition between these phases. The main experimental features of the $C s D_{1}$ and $D_{2}$ spectra in liquid, solid bcc, and solid hcp helium are collected in Tables 1 and 2. 


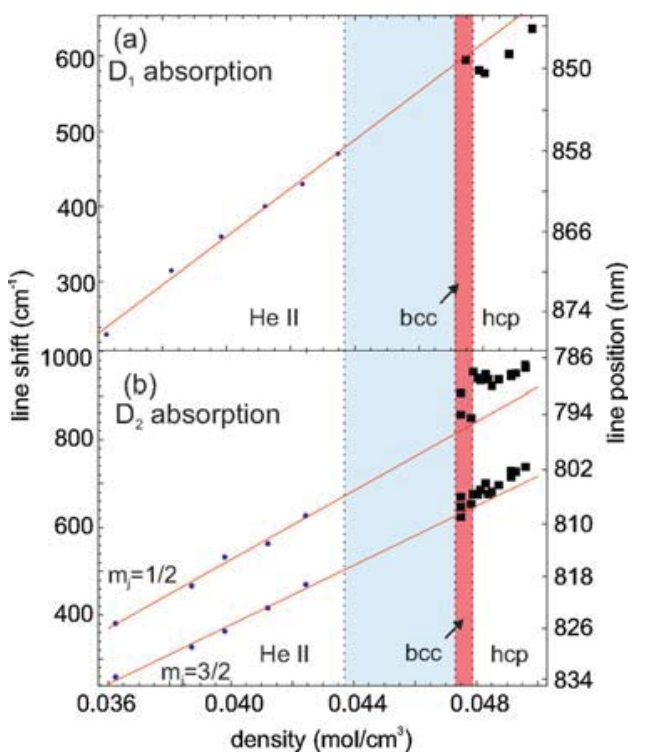

Figure 5. Dependence of the $\mathrm{D}_{1}$ (a) and $\mathrm{D}_{2}$ (b) excitation line wavelength and their shifts (left scale) with respect to the free Cs atom on the liquid and solid helium density at $T=1.5 \mathrm{~K}$. Data in liquid He are taken from ref 28; data in solid He are obtained in the present work. The red solid line fits the experimental data points in the liquid. The pink color highlights the region of bcc solid $\mathrm{He}$, and the light-blue colored region refers to the nonaccessible conditions corresponding to the density jump at the liquid-solid transition.

The density dependence of the line positions shown in Figure 5 seems to indicate that the doublet splitting of the $D_{2}$ line observed earlier ${ }^{20}$ in liquid He smoothly extends into the bcc phase, suggesting a similar mechanism of the splitting in both phases. At the bcc-hcp phase transition the doublet splitting shows a step-like change. The blue component in hcp is blueshifted by $4 \mathrm{~nm}$ with respect to bcc, which is comparable to the total splitting of $15 \mathrm{~nm}$, while the position of the red component is almost the same in both solid phases.

\section{THEORETICAL MODEL}

Recently we have presented results of spherical bubble model (SBM) calculations of the $\mathrm{D}_{1}\left(6 S_{1 / 2}-6 P_{1 / 2}\right)$ transition of Cs atom in solid helium. ${ }^{13}$ Appendix A gives a short review of that model.

The theoretical predictions of the line positions by the SBM differ from experimental results by several $\mathrm{cm}^{-1}$ in case of the $D_{1}$ transition (both in absorption and emission) and cannot account for the doublet splitting of the $\mathrm{D}_{2}$ line. In the present work we try to attribute the observed discrepancies between theoretical predictions and experimental results to the anisotropy of the elastic properties of the solid He matrix.

The development of a deformed bubble model (DBM) that includes the anisotropic elasticity of the medium, and the anisotropic $\mathrm{Cs}-\mathrm{He}$ interaction arising from it, encounters several principal and technical problems. While the SBM is basically a one-dimensional problem because it involves only a radial coordinate, any description of bubble deformations involves truly three-dimensional calculations, which makes the explicit formulation of the DBM and its numerical solution a demanding problem. Kinoshita et al. ${ }^{28}$ have used an alternative approach that accounted for the helium bubble deformation by a nonspherical

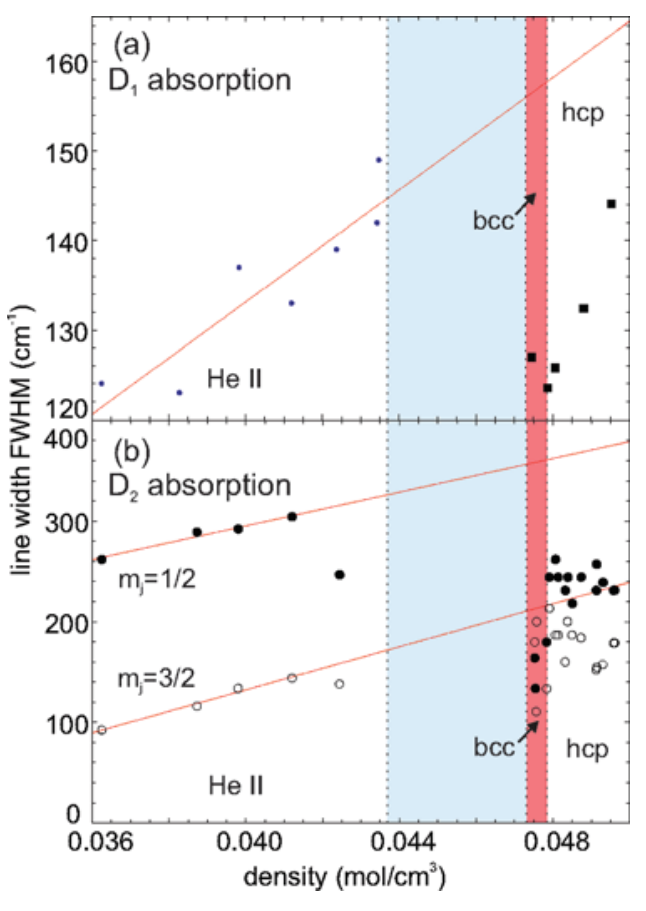

Figure 6. Dependence of the $\mathrm{D}_{1}(\mathrm{a})$ and $\mathrm{D}_{2}$ (b) excitation line widths (fwhm) on the liquid and solid helium density at $T=1.5 \mathrm{~K}$. Data in liquid $\mathrm{He}$ are taken from ref 28; data in solid $\mathrm{He}$ are obtained in the present work. The red solid line fits the experimental data points in the liquid. The pink color highlights the region of bcc solid $\mathrm{He}$, and the light-blue colored region refers to the nonaccessible conditions corresponding to the density jump at the liquid-solid transition.

helium density distribution, in combination with effective radial cesium-helium interaction potentials. That approach is equivalent to a first order perturbation calculation, and we will discuss the applicability of that approach for He solids.

The SBM allows us to calculate the energy eigenstates of the $\mathrm{Cs}$ atom valence electron in a homogeneous and isotropic $\mathrm{He}$ environment. The differences between the measured and calculated transition energies are small compared to the energy shifts induced by the He matrix (compared to free atom).

Because of the very small density difference between bcc and hcp matrices, the SBM does not explain the significant jump of the transition wavelengths observed at the bcc-hcp phase transition. Moreover, the linewidths predicted by this model are smaller than those measured, both for excitation and emission lines (cf. Table 2).

The above approach does not allow us to account for the plastic deformation of the matrix during freezing. We try to build a model that incorporates the well-known equilibrium elastic properties of helium crystals. We start by replacing the energy term based on the balance consideration in the total bubble energy expression (eq 28 of Appendix A) by a volumetric elastic energy defined as

$$
E_{\text {vol.el. }}\left(R_{\mathrm{b}}, \varepsilon\right)=\beta\left(V-V_{0}\right)
$$

where $\beta$ is the bulk elastic modulus, $V$ the effective volume of the bubble, and $V_{0}$ the volume of the bubble at zero elasticity condition, that is, obtained by ignoring the last term in eq 27.

The volumetric elastic constants were determined for the bcc and hcp helium crystalline phases by various methods (see, e.g., $\left.{ }^{7,8,29-31}\right)$. Similar values of $3-4 \times 10^{7} \mathrm{~Pa}$ were obtained 
for bcc and hcp, and the volumetric energy for a given bubble size is on the order of several wavenumbers. It is, therefore, not surprising that the SBM, including the volumetric elasticity, predicts practically the same line positions for the excitation and emission $\mathrm{D}_{1}$ lines of $\mathrm{Cs}$ in both bcc and hcp matrices. The line positions, thus, do not differ much from the ones predicted by the SBM calculations presented in ref 13 . However, the predicted linewidths are found to be in a better agreement with the experimental results (cf. Table 2). Therefore, the inclusion of volumetric elasticity improves the present calculation compared to the SBM calculation presented in ref 13.

5.1. Pair Potentials Approach in Deformation Modeling. The anisotropic interaction between the $\mathrm{He}$ matrix and the Cs atom can be described by a model based on $\mathrm{Cs}-\mathrm{He}$ interaction

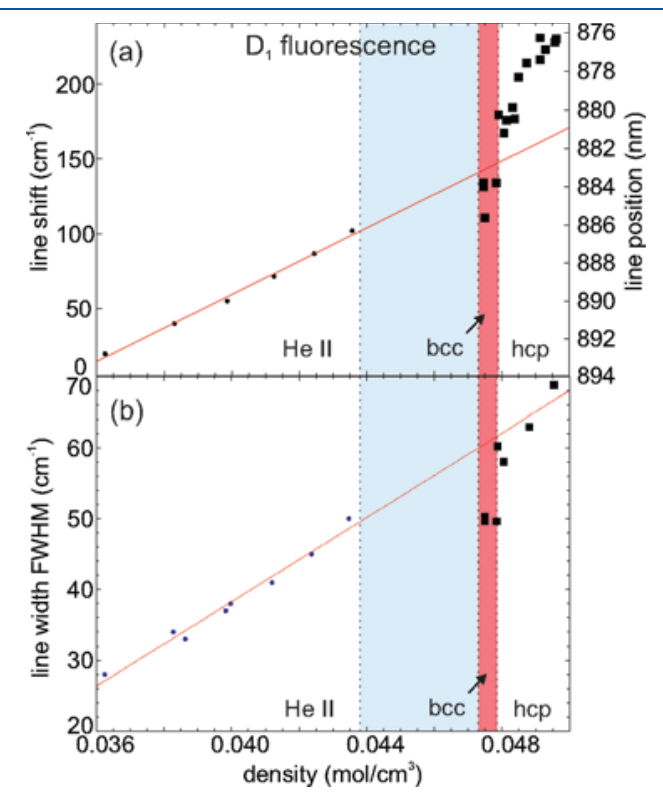

Figure 7. Dependence of the $D_{1}$ fluorescence wavelength and its shift with respect to the free Cs atom (a) and line width (b) on the liquid and solid helium density at $T=1.5 \mathrm{~K}$. Data in liquid He are taken from ref 28; data in solid $\mathrm{He}$ are obtained in the present work. The red solid line fits the experimental data points in the liquid. The pink color highlights the region of bcc solid $\mathrm{He}$, and the light-blue colored region refers to the nonaccessible conditions corresponding to the density jump at the liquid-solid transition. pair potentials, as given, for example, in ref 32. As zero-order approximation we use, in the frame of the SBM, the deformation induced by an isotropic bulk solid on the Cs atom. The force applied to an infinitely small element of the bubble boundary can be derived from the potential of the Cs-He interaction and the bubble parameters given by the SBM. This force must be equilibrated by the anisotropic elastic response from the medium. In Appendix B we discuss the generalization of Hooke's law for anisotropic elastic media. We use the results of eq 30 to calculate the strain for different points on the bubble interface. The resulting radial profile is then a first approximation to the deformed atomic bubble shape in the anisotropic medium. We iteratively apply this procedure to the deformed bubbles, until the bubble deformation converges to a stable shape.

In the hcp matrix, the iterative procedure yields an ellipsoidal bubble shape with main axes whose lengths differ by $\approx 7 \% .{ }^{5} \mathrm{We}$ were unable to make the described procedure converge for the bcc crystal, presumably due to its very pronounced anisotropy (Figure 11).

The atomic energy levels calculated in this way lead to blue shifts of the excitation lines which exceed the measured shifts by typically $500 \mathrm{~cm}^{-1}$. We suspect that the origin of this discrepancy lies in the insufficient precision of the long-range parts of the available pair potentials. The pair potential approach does not produce reliable results, while the perturbative approach, presented in the next paragraph yields a much better description of the experimental observations.

5.2. Anisotropic Perturbation Hamiltonian. In the SBM calculation, the assumption of an isotropic elastic response of the solid He matrix does not reproduce the measured spectral line shifts. We have therefore extended the SBM to deformed bubbles by assuming that the bubble deformation has the same symmetry as the anisotropic compressibility $\kappa(\mathbf{n})$ of the matrix. The explicit form of $\kappa(\mathbf{n})$ is given in Appendix B. We assume (as justified by the results of the calculation) that the bubble deformations are small compared to the average bubble size, so that we can apply a perturbative treatment to the problem. We use the electronic eigenfunctions of the SBM as an orthonormal basis set for the zero-order unperturbed wave functions. Calculations were carried out for the $6 S_{1 / 2}$ ground state and the first excited $6 P_{1 / 2}$ and $6 P_{3 / 2}$ states.

The interaction Hamiltonian of the Cs atom and the deformed bubble is

$$
V_{\text {def }}(r)=V_{\text {free }}(r)+V_{\mathrm{He}}^{\mathrm{def}}(r)
$$

Table 1. Measured and Calculated Wavelengths of the D-Line Centers of Cs in Condensed He at $1.5 \mathrm{~K}$

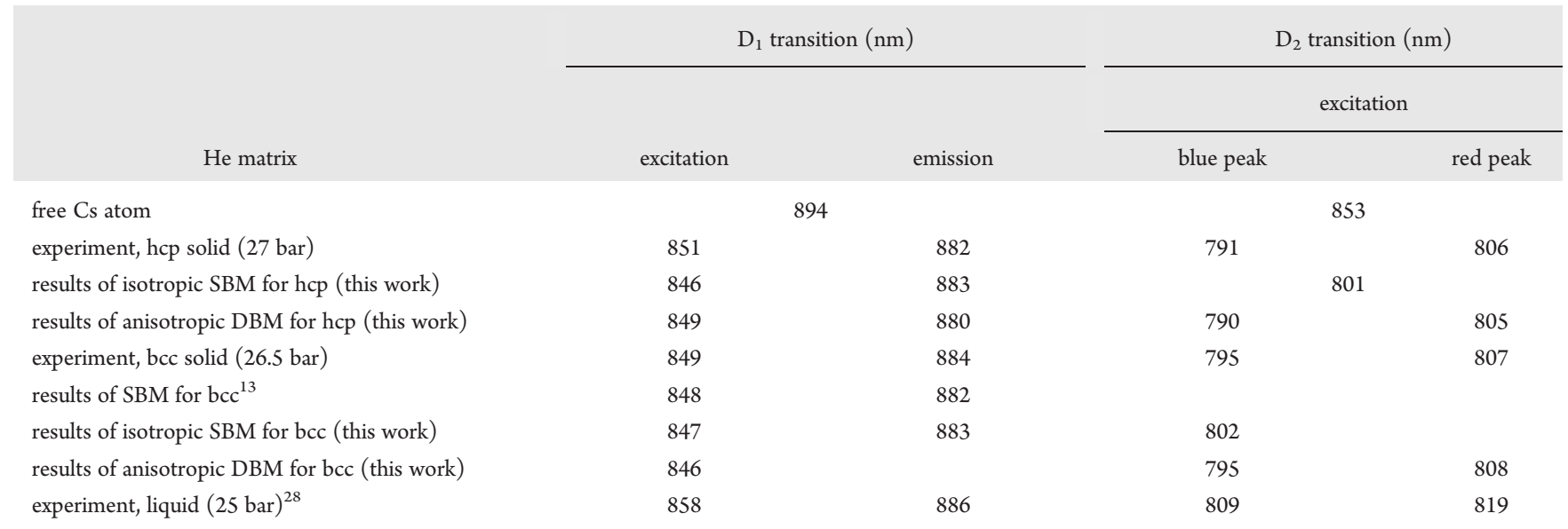


Table 2. Measured and Calculated Linewidths (Full Widths at Half Maximum) of the D-Lines of Cs in Condensed He at $1.5 \mathrm{~K}$

\begin{tabular}{|c|c|c|c|c|}
\hline \multirow[b]{3}{*}{ He matrix } & \multicolumn{2}{|c|}{$\mathrm{D}_{1}$ transition $\left(\mathrm{cm}^{-1}\right)$} & \multicolumn{2}{|c|}{$\mathrm{D}_{2}$ transition $\left(\mathrm{cm}^{-1}\right)$} \\
\hline & \multirow[b]{2}{*}{ excitation } & \multirow[b]{2}{*}{ emission } & \multicolumn{2}{|c|}{ excitation } \\
\hline & & & blue peak & red peak \\
\hline experiment, hcp solid (27 bar) & 152 & 77 & 180 & 125 \\
\hline results of isotropic SBM for hcp (this work) & 180 & 70 & & \\
\hline experiment, bcc solid (26.5 bar) & 180 & 70 & 160 & 125 \\
\hline results of SBM for bcc ${ }^{13}$ & 125 & 40 & & \\
\hline results of isotropic SBM for bcc (this work) & 170 & 65 & & \\
\hline experiment, liquid $(25 \mathrm{bar})^{20}$ & 135 & 50 & 240 & 150 \\
\hline
\end{tabular}

where the pseudopotential in the deformed bubble is given by

$$
V_{\mathrm{He}}^{\mathrm{def}}(r)=\iint \rho^{\mathrm{def}}(\mathbf{R}) V_{\mathrm{He}}(\mathbf{r}, \mathbf{R}) \mathrm{d} \mathbf{R} \mathrm{d} \Omega_{\mathrm{e}}
$$

We parametrize the helium density distribution in the deformed bubble according to eq 25 , in which we give $R_{0}$ an angular dependence expressed in terms of spherical harmonics

$$
R_{0}(\theta, \phi)=R_{0}\left(1+\sqrt{4 \pi} \sum_{l, m} B_{l, m}^{\mathrm{cryst}} Y_{m}^{(l)}(\theta, \phi)\right)
$$

where the coefficients $B_{l, m}^{\text {cryst }}$ characterize the bubble deformation in a given $\mathrm{He}$ crystal. The change of the $\mathrm{Cs}-\mathrm{He}$ interaction energy due to the bubble deformation is then given by

$$
\begin{aligned}
\Delta V(\mathbf{r}) & =V_{\mathrm{He}}^{\mathrm{def}}(\mathbf{r})-V_{\mathrm{He}}^{\mathrm{sph}}(\mathbf{r}) \\
& =\iint\left[\rho^{\mathrm{def}}(\mathbf{R})-\rho^{\mathrm{sph}}(\mathbf{R})\right] V_{\mathrm{He}}(\mathbf{r}, \mathbf{R}) \mathrm{d} \mathbf{R}
\end{aligned}
$$

where we have assumed that contribution from $V_{\text {free }}$ in eq 2 does not depend on the bubble deformation.

The energy change of eq 5 can be factorized into radial and angular parts according to

$$
\Delta V(r, \theta, \phi)=\mathscr{R}_{\text {cryst }}(r) \mathscr{l}_{\text {cryst }}(\theta, \phi)
$$

where

$$
\mathcal{A}_{\text {cryst }}(\theta, \phi)=B_{0,0}^{\text {cryst }} Y_{0}^{(0)}(\theta, \phi)+\sum_{l>0, m} B_{l, m}^{\text {cryst }} Y_{m}^{(l)}(\theta, \phi)
$$

and where the coefficients $B_{0,0}^{\text {cryst }}$ and $B_{l, m}^{\text {cryst }}$ represent the changes of the bubble size and the bubble deformation induced by the specific crystal structure. In eqs 6 and 7, we have assumed that the bubble deformation is small compared to the interface width of the bubble (given by $\varepsilon^{-1}$ ), which has allowed us to neglect the angular dependence of $R_{0}$ in the exponential factor of the modified density profile $\rho^{\text {def }}$. For the hcp matrix eq 7 yields

$$
\mathcal{A}_{\mathrm{hcp}}(\theta, \phi)=B_{0,0}^{\mathrm{hcp}} Y_{0}^{(0)}+B_{2,0}^{\mathrm{hcp}} Y_{0}^{(2)}+B_{4,0}^{\mathrm{hcp}} Y_{0}^{(4)}
$$

while for the bcc matrix one finds

$$
\mathcal{A}_{\mathrm{bcc}}(\theta, \phi)=B_{0,0}^{\mathrm{bcc}} Y_{0}^{(0)}+B_{4,0}^{\mathrm{bcc}} Y_{0}^{(4)}+B_{4, \pm 4}^{\mathrm{bcc}}\left(Y_{4}^{(4)}+Y_{-4}^{(4)}\right)
$$

with $B_{4, \pm 4}^{\mathrm{bcc}} / B_{4,0}^{\mathrm{bcc}}=1 / 2(10 / 7)^{1 / 2}$.

The leading deformation terms thus have quadrupolar $(l=2)$ and hexadecupolar $(l=4)$ symmetries for the hcp and bcc phase, respectively. We note that the deformation potentials proportional to $Y_{0}^{(0)}$ describe changes of the bubble volume.

5.3. Matrix Elements. By writing the atomic wave functions as

$$
\left|\Psi_{\alpha}\right\rangle \equiv\left|n_{\alpha}, L_{\alpha}, J_{\alpha}, M_{J_{\alpha}}\right\rangle \equiv|\alpha\rangle
$$

one can factorize the perturbation operator matrix elements according to

$$
\langle\beta|\Delta V(\mathbf{r})| \alpha\rangle=I_{\mathscr{R}}^{(\alpha, \beta)} I_{\Omega}^{(\alpha, \beta)}
$$

where the radial integral

$$
I_{\mathscr{R}}^{\alpha, \beta}=\int R_{\beta}(r) \mathscr{R}_{\text {cryst }}(r) R_{\alpha}(r) r^{2} \mathrm{~d} r
$$

is evaluated numerically, $R_{\alpha}(r)$ and $R_{\beta}(r)$ being the radial wave functions. The angular integral is given by

$$
I_{\Omega}^{(\alpha, \beta)}=\left\langle\beta\left|\mathcal{A}_{\text {cryst }}(\theta, \phi)\right| \alpha\right\rangle
$$

Because $\mathcal{A}_{\text {cryst }}$ is expressed in terms of spherical harmonics by eqs 8 and 9, the matrix elements in eq 13 are readily evaluated using the Wigner-Eckart theorem and angular momentum decoupling rules (cf., e.g., ref 33), yielding

$$
\begin{aligned}
& I_{\Omega}^{(\alpha, \beta)}= \\
& (4 \pi)^{-1 / 2}(-1)^{J_{\alpha}-M_{J_{\alpha}}+1 / 2+J_{\beta}} \sqrt{\left(2 J_{\alpha}+1\right)\left(2 J_{\beta}+1\right)\left(2 L_{\alpha}+1\right)\left(2 L_{\beta}+1\right)} \\
& \quad \times \sum_{l, m}(-1)^{l}\left(\begin{array}{ccc}
J_{\alpha} & l & J_{\beta} \\
-M_{J_{\alpha}} & m & M_{J_{\beta}}
\end{array}\right)\left(\begin{array}{ccc}
L_{\alpha} & l & L_{\beta} \\
0 & 0 & 0
\end{array}\right)\left\{\begin{array}{ccc}
L_{\alpha} & J_{\alpha} & \frac{1}{2} \\
J_{\beta} & L_{\beta} & l
\end{array}\right\}
\end{aligned}
$$

where the round (curly) brackets denote $3 j(6 j)$ symbols. The sum in eq 14 is carried out over the specific multipole moments over the phase under consideration.

The summation of the magnetic quantum numbers $m$ shows that the $Y_{ \pm 4}^{(4)}$ terms in eq 9 give no net contribution, so that the only nonvanishing contributions come from the $m=0$ terms of the multipole expansion. In the hcp phase, the contribution from the $Y_{0}^{(4)}$ term turns out to be small compared to the contribution from the $Y_{0}^{(2)}$ term and is thus neglected. The angular factors $\mathcal{A}_{\text {cryst }}(\theta, \phi)$ are thus well approximated by

$$
\mathscr{C}_{\mathrm{hcp}}(\theta, \phi) \approx B_{0,0}^{\mathrm{hcp}} Y_{0}^{(0)}+B_{2,0}^{\mathrm{hcp}} Y_{0}^{(2)}
$$

and

$$
\mathcal{C}_{\mathrm{bcc}}(\theta, \phi) \approx B_{0,0}^{\mathrm{bcc}} Y_{0}^{(0)}+B_{4,0}^{\mathrm{bcc}} Y_{0}^{(4)}
$$


Table 3. Electronic States of the Cs Atom Mixed by the Interaction with the hcp and bcc Helium Matrices in Second Order Perturbation Theory

\begin{tabular}{ccc} 
matrix & \multicolumn{1}{c}{ hcp } & \multicolumn{1}{c}{ bcc } \\
perturbed state & \multicolumn{1}{c}{ admixed state } \\
\cline { 2 - 3 } $6 S_{1 / 2}$ & $n S_{1 / 2}, n D_{3 / 2,5 / 2}$ & \multicolumn{1}{c}{$n S_{1 / 2}, n G_{7 / 2,9 / 2}$} \\
$6 P_{1 / 2}$ & $n P_{1 / 2,3 / 2}, n F_{5 / 2}$ & $n F_{7 / 2}$ \\
$6 P_{3 / 2}$ & $n P_{3 / 2}, n F_{5 / 2,7 / 2}$ & $n F_{5 / 2,7 / 2}$ \\
\hline
\end{tabular}

meaning that the sum in eq 14 runs over $(1, m)=(0,0)$ and $(2,0)$ for hcp crystals, while in bcc it sums over $(l, m)=(0,0)$ and $(4,0)$.

The selection rules of the $3 j$ and $6 j$ symbols imply that in hcp the interaction of eq 15 admixes $n S_{1 / 2}, n D_{J}$ states to the $6 S_{1 / 2}$ state, and $n P_{J}, n F_{J}$ states to the $6 P_{J}$ states. In bcc, the interaction of eq 16 admixes $n S_{1 / 2}, n G_{J}$ states to the $6 S_{1 / 2}$ state, and $n P_{J}, n F_{J}$, and $n H_{J}$ states to the $6 P_{J}$ states. The numerical evaluation of the radial integrals shows that the contribution from $n H_{J}$ states is negligible. Table 3 summarizes the state admixtures taken into account in our calculations.

5.4. Perturbed Energies. In first order, the energy perturbations are given by

$$
E_{\alpha}^{(1)}=\left\langle\Psi_{\alpha}^{(0)}|\Delta V(\mathbf{r})| \Psi_{\alpha}^{(0)}\right\rangle
$$

For $n S_{1 / 2}$ states, only the matrix elements of $Y_{0}^{(0)}$ give a nonvanishing contribution, while for the $n P_{J}$ states, only the matrix elements $\left\langle n P_{J}\left|Y_{0}^{(0)}\right| n P_{J}\right\rangle$ and $\left\langle n P_{3 / 2}, M_{J}\left|Y_{0}^{(2)}\right| n P_{3 / 2}, M_{J}\right\rangle$ contribute. The latter splits the $n P_{3 / 2}$ state into two components with $\left|M_{J}\right|=$ $1 / 2$ and $\left|M_{J}\right|=3 / 2$.

In second order, the energy perturbations are given by

$$
E_{i}^{(2)}=\sum_{j \neq i} \frac{\left|\left\langle\Psi_{j}|\Delta V(\mathbf{r})| \Psi_{i}\right\rangle\right|^{2}}{E_{i}^{(0)}-E_{j}^{(0)}}
$$

In bcc and hcp, the $Y_{0}^{(0)}$ term mixes states of the same angular momentum, which shifts the $6 S_{1 / 2}$ and $6 P_{J}$ states to lower energies.

In hcp, the $Y_{0}^{(2)}$ interaction mixes the $6 S_{1 / 2}$ state with $n D_{J}$ states which down-shifts its energy. The same term induces a J-mixing of the two $6 P_{J}$ states, thus, giving the main contribution to the spectral shifts of the $\mathrm{D}_{1}$ and $\mathrm{D}_{2}$ lines.

In bcc, the $6 S_{1 / 2}$ is mixed with $n G_{J}$ states due to the $Y_{0}^{(4)}$ term. Its energy remains practically unchanged because of the small value of the corresponding radial integral. The main perturbation of the $6 P_{J}$ states comes from $n F$ admixtures, which also lifts the degeneracy of the $6 P_{3 / 2}$ state.

Third order corrections were found to be very small in comparison to the second order effects.

Because our experimental resolution does not allow us to resolve their effect, they will be neglected.

5.5. Determination of the Coefficients $B_{l, m}$. The state energies, $E_{n L}$, are represented by surfaces in the space spanned by the coefficients $B_{l, m}^{\text {cryst }}$. The experiments yield transition wavelengths, $\lambda_{n L}^{\exp }$, whose corresponding energies are represented by horizontal planes in that parameter space. With the approximations introduced above, that space reduces to two dimensions: $E_{n L_{J}}\left(B_{0,0}^{\mathrm{bcc}}, B_{4,0}^{\mathrm{bcc}}\right)$ for bcc and $E_{n L_{I}}\left(B_{0,0}^{\mathrm{hcp}}, B_{2,0}^{\mathrm{hcp}}\right)$ for hcp. We determined the optimal parameters $B_{l, m}^{\text {cryst }}$ by a numerical minimization of the squared deviations

$$
\mathscr{M}\left(B_{l, m}^{\text {cryst }}\right)=\sum_{J,\left|M_{J}\right|}\left[\lambda_{J,\left|M_{J}\right|}^{\text {theo }}\left(B_{l, m}^{\text {cryst }}\right)-\lambda_{J,\left|M_{J}\right|}^{\text {exp }}\right]^{2}
$$

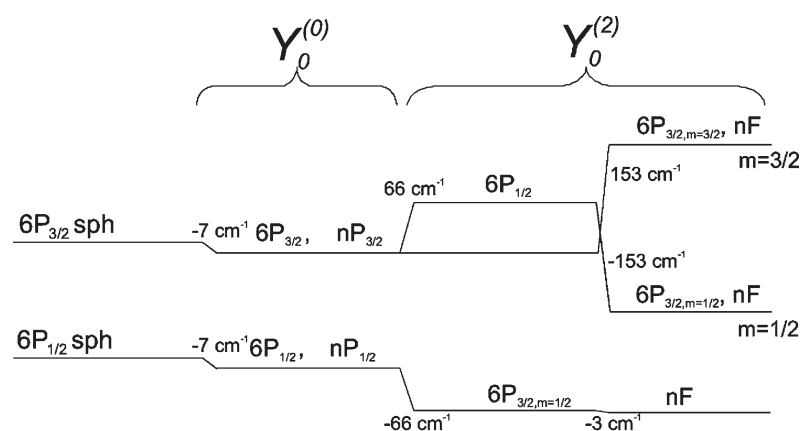

$6 \mathrm{~S}_{1 / 2} \mathrm{sph}$ $-2 \mathrm{~cm}^{-1} 6 \mathrm{~S}_{1 / 2}, \quad \mathrm{nS}_{1 / 2}$ $-2 \mathrm{~cm}^{-1} \quad \mathrm{nD}$

Figure 8. Shifts of the $6 S_{1 / 2}$ and $6 P_{1 / 2,3 / 2}$ energy levels of Cs due to state mixing in the hcp bubble. SBM energies are shown on the left. The contributions from $Y_{0}^{(0)}$ and $Y_{0}^{(2)}$ terms of $\Delta V$ are shown separately. The electronic states that are admixed by each term are indicated next to the energy level. Numerical values correspond to the bubble configuration that provides the best fit of the experimental data.

between experimental and theoretical values of the transition wavelength, where

$$
\lambda_{J,\left|M_{J}\right|}^{\text {theo }}\left(B_{l, m}^{\text {cryst }}\right)=\frac{h c}{\tilde{E}_{6 P_{J},\left|M_{J}\right|}-\tilde{E}_{6 S_{1 / 2}}}
$$

and

$$
\tilde{E}_{n L_{J},\left|M_{J}\right|}=E_{n L_{J},\left|M_{J}\right|}^{(0)}+E_{n L_{J},\left|M_{J}\right|}^{(1)}+E_{n L_{J},\left|M_{J}\right|}^{(2)}
$$

The $\lambda_{J,\left|M_{J}\right|}^{\exp }$ are the measured transition wavelengths from the $6 S_{1 / 2}$ ground state to the excited $\left|6 P_{J},\right| M_{J}|\rangle$ states as given in Table 1. Figure 9 shows cuts through the hcp and bcc energy surfaces for $B_{0,0}^{\text {cryst }}=0$ (dotted lines) and for $B_{0,0}^{\text {cryst }}$ given by the best fit values (solid lines). The graphs in Figure 9 show a sign ambiguity (prolate vs oblong) of the deformation, which can be resolved by a physical argument. Based on the directional dependence of the compressibility, not explicitly used in this perturbative treatment, we can unambiguously determine the sign of the deformation. We expect the bubble in hcp to be compressed along the $c$-axis (Figure 11), which corresponds to a negative value of $B_{2,0}^{\text {hcp }}$. In bcc we expect the bubble to be stretched along the three crystallographic axes, thus taking a hexoctahedronal shape, which corresponds to a positive value of $B_{4,0}^{\mathrm{bcc}}$. The respective bubble shapes obtained by the minimization are shown (to scale) in Figure 10 for the deformation parameter values listed in Table 4.

The above discussion has addressed only the deformation of ground state bubbles and is, thus, well suited to account for the excitation process in which the bubble shape and size do not change (Franck-Condon principle). The corresponding treatment of the emission process requires a calculation of deformed excited state bubbles. Because there is no emission on the $\mathrm{D}_{2}$ line, the only experimental value is the $\mathrm{D}_{1}$ emission wavelength, which is not sufficient to infer the two free parameters.

\section{DISCUSSION}

The fit of the experimental data presented in the previous section allows us to obtain the configuration of the atomic bubble formed around a ground state Cs atom in solid helium. This 


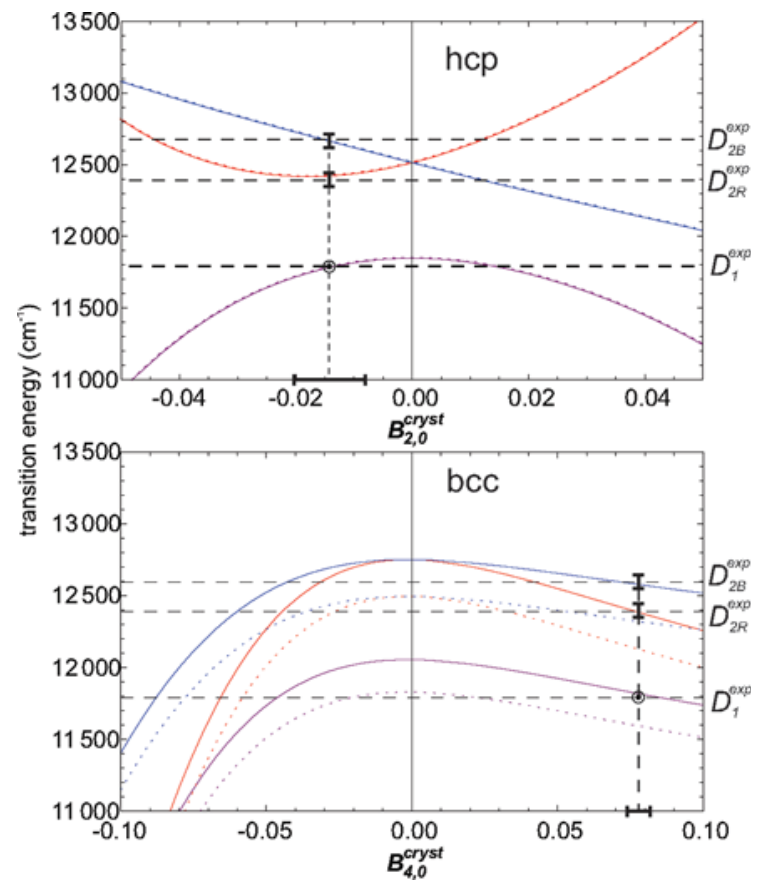

Figure 9. Dependence of the transition energies on the bubble deformation parameters for the hcp (top) and for the bcc (bottom) phases. Solid lines are obtained with $B_{0,0}^{\text {cryst }}$ corresponding to the best fit bubble configuration. (Dotted lines corresponding to a configuration with $B_{0,0}^{\text {cryst }}=0$ are indistinguishable from the solid lines in hcp.) The horizontal dashed lines show the experimental transition energies (with the experimental error bars). Vertical dashed lines indicate the best fit configurations.

Table 4. Changes of Bubble Size and Deformation, as Compared to the Spherical Bubble Model Prediction, Obtained from Fits to Experimental Transition Wavelengths

\begin{tabular}{lll} 
& \multicolumn{1}{c}{$B_{0,0}^{\text {cryst }}$} & \multicolumn{1}{c}{$B_{l>0, m}^{\text {cryst }}$} \\
\hline hcp solid & $0(7) \times 10^{-3}$ & $-14(6) \times 10^{-3}$ \\
bcc solid & $+15(4) \times 10^{-3}$ & $+80(14) \times 10^{-3}$ \\
\hline
\end{tabular}

configuration should correspond to a minimum of the total (atom plus bubble) energy given by eqs 26 and 27 that lies slightly below the energy of the atom in the spherical bubble (a direct minimization of eq 26 is technically not feasible). In the Born-Oppenheimer approximation, the excitation of the Cs atom by photon absorption occurs in this bubble configuration. Because the electron density distribution of the excited $6 P_{J}$ state does not fit well into this relatively small bubble, the excited atom is strongly perturbed and its energy levels shift by several $100 \mathrm{~cm}^{-1}$, depending on the bubble deformation (Figure 9).

The modifications of the Cs atom energy levels in the hcp phase are schematically summarized in Figure 8. Compared to the SBM, the $6 S_{1 / 2}$ and $6 P_{1 / 2}$ energies are down-shifted by 4 and $76 \mathrm{~cm}^{-1}$, respectively. The $6 P_{3 / 2}$ level is split into two components, one of which $\left(\left|M_{J}\right|=3 / 2\right)$ is upshifted by $146 \mathrm{~cm}^{-1}$, while the other $\left(\left|M_{J}\right|=1 / 2\right)$ is down-shifted by $94 \mathrm{~cm}^{-1}$ with respect to the SBM predictions. The observed Cs atom absorption spectra in hcp solid helium are thus successfully described by our theoretical model assuming a small static deformation $\left(R_{\max }-R_{\min }\right) /\left(R_{\max }+R_{\min }\right)=1.6 \%$ of the bubble, whose symmetry corresponds to that of the anisotropic stiffness of the matrix.
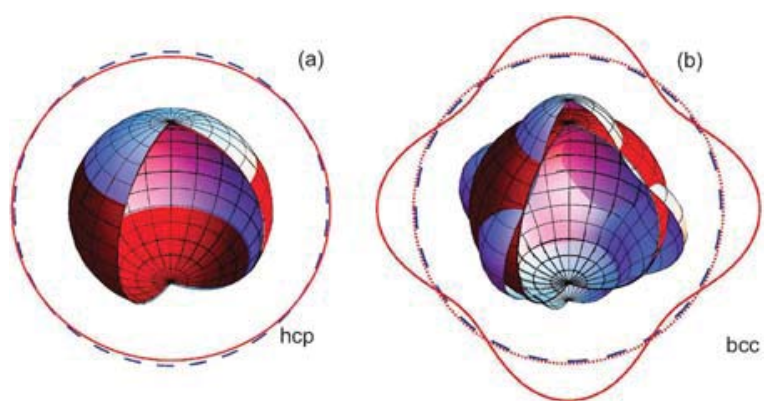

Figure 10. Cross section of the deformed bubble (solid red curves). The comparison with spherical bubble (dashed blue curves) in hcp (a) and bcc (b) phases of solid He. Dotted curve in (b) represents the isotropic expansion contribution; for hcp it is indistinguishable from the original bubble shape.

This can be compared with the results of a calculation ${ }^{5}$ utilizing Cs-He pair potentials (described in Subsection 5.1) that gives a deformation of $3.5 \%$ and with the results of an analysis of the hyperfine spectra ${ }^{16}$ that yielded a deformation of $3.5 \%$.

When applied to the bcc matrix, the same model suggests a much stronger bubble deformation of $18 \%$. The resulting bubble shape shown in Figure 10b indeed differs significantly from spherical. Besides changing its shape, the bubble also becomes slightly larger due to the positive $B_{0,0}^{\text {hcp }}$ term (Table 4). This increase of the bubble volume and interface area leads to an increase of $7 \mathrm{~cm}^{-1}$ in the surface energy and $p V$ terms in eq 27. At the same time, the electronic energy, $E_{\text {eig, }}$ of the ground state is reduced by $7 \mathrm{~cm}^{-1}$. The bubble deformation reduces the elastic term in eq 27 and therefore the total (atom plus bubble) energy becomes lower than for the spherical bubble. Unfortunately, we could not, as yet, calculate the elastic energy and bubble deformation from first principles.

As one can see in Figures 5 and 7, the density dependence of the lineshifts has no discontinuity at the liquid-bcc phase boundary (when the small density difference between the two phases is properly taken into account). It seems likely that the lineshifts and the splitting of the $\mathrm{D}_{2}$ line in bcc have the same origin as in liquid helium. The linear increase of the lineshift with helium density is successfully explained by the spherical bubble model. ${ }^{13,28}$ On the other hand, to explain the $\mathrm{D}_{2}$ splitting, one has to postulate a nonspherical bubble shape. It was pointed out by Kinoshita et al. $^{20}$ that quadrupole oscillations of the bubble interface in superfluid helium may explain this splitting. However, their calculated splittings and linewidths strongly underestimate the observed effect.

Bcc solid and superfluid helium matrices perturb the electron spins of embedded Cs atoms only weakly, in strong contrast with hcp crystals. This is evidenced by the extremely long spin depolarization time ${ }^{26}$ and the very narrow magnetic resonance spectra (hyperfine and Zeeman) in HeII and bcc-He. ${ }^{16}$ It was therefore suggested in ref 16 that the atomic bubbles in bcc are spherically symmetric and that a static bubble deformation exists only in hcp. On the other hand, as was noticed in, 5 even in a strongly deformed bubble, the coupling between the bubble deformation and the electron spin depends on the multipolarity of the deformation symmetry and may be very weak. In the bcc matrix, the $6 S_{1 / 2}$ state of Cs is mixed with $n S_{1 / 2}$ and $n G_{7 / 2,9 / 2}$ states (Table 3 ) due to the hexadecupolar interaction. The effect 
of such perturbation on the hyperfine and Zeeman structure of the $6 S_{1 / 2}$ state is expected to be strongly suppressed. ${ }^{5}$

Despite extensive experimental and theoretical studies of atomic bubbles in condensed helium, static and dynamic bubble deformations still pose some open questions, in particular, for the bcc phase. A successful model should be able to predict both optical and ODMR spectra in all three phases by combining the present static bubble deformation analysis with the effects of dynamic bubble distortions (vibrations). We also plan to apply the formalism presented here for modeling magnetic resonance spectra in bcc and hcp matrices.

\section{SUMMARY}

We have studied experimentally and theoretically the optical spectra of the $\mathrm{D}_{1}$ and $\mathrm{D}_{2}$ transitions of $\mathrm{Cs}$ atoms trapped in (anisotropic) hcp and bcc crystalline matrices of ${ }^{4} \mathrm{He}$. We have calculated the energies of the three lowest-lying Cs states by numerically solving the Schrödinger equation using a ThomasFermi potential for the Cs valence electron and pseudopotentials that describe the interaction of the Cs valence electron and the Cs core with the helium atoms. We presented results for spherical bubbles and have extended the model to deformed bubbles, for which we performed a perturbative calculation of the energy modifications induced by anisotropic helium distributions. We have successfully reproduced the observed spectra in both crystalline phases of $\mathrm{He}$, after fitting our two-parameter model predictions. For the hcp matrix we have obtained a small quadrupolar bubble deformation with a negligible volume change, whereas in bcc, we find a larger hexadecupolar deformation and a finite volume change. For hcp matrices, the results agree (within a factor of 2 ) with earlier treatments based on the analysis of experimental ODMR spectra ${ }^{16}$ and on the known macroscopic elastic properties of helium crystals. ${ }^{5}$ The unexpected large deformation obtained for bcc matrices needs further investigations. The experimental results obtained in bcc can also be explained (at least qualitatively) by a bubble vibration model, developed earlier ${ }^{20}$ for superfluid helium, and future studies will need to treat static and dynamic deformations in a consistent manner.

\section{APPENDIX A. SPHERICAL BUBBLE MODEL}

The spherical bubble model describes the atomic bubble as consisting of the impurity atom surrounded by a homogeneous isotropic distribution of helium with known (macroscopic) parameters, namely, the density, $\rho$, and the surface tension, $\sigma$. The multielectron alkali atom proper is modeled as a valence electron moving in the effective Thomas-Fermi potential formed by the nucleus and the $Z-1$ core electrons. Dipolar and quadrupolar polarization interactions of the valence electron with the core and spin-orbit interaction are included as corrections.

The energy eigenstates of the atomic bubble are determined by the Schrödinger equation

$$
\Delta \Psi^{\mathrm{sph}}(\mathbf{r})+2\left[E-V_{\mathrm{sph}}(r)-\frac{l(l+1)}{2 r^{2}}\right] \Psi^{\mathrm{sph}}(\mathbf{r})=0
$$

written in atomic units. Here

$$
V_{\text {sph }}(r)=V_{\text {free }}(r)+V_{\mathrm{He}}^{\mathrm{sph}}(r)
$$
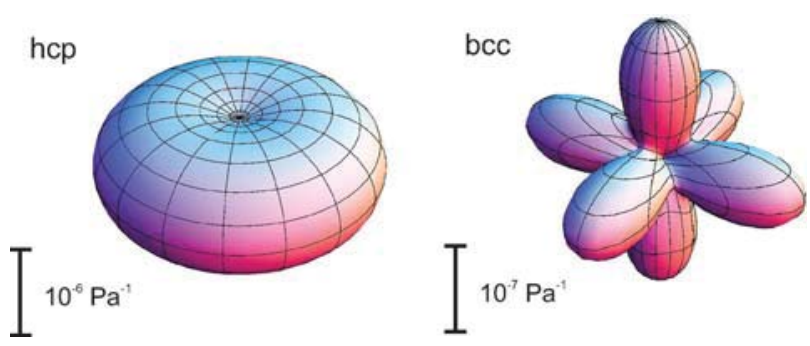

Figure 11. Directional diagram of the compressibilities $\kappa$ of the hcp (left) and bcc (right) crystalline lattices of ${ }^{4} \mathrm{He}$.

is the potential seen by the valence electron. The potential seen by the valence electron in the free atom is given by

$$
V_{\text {free }}(r)=V_{\mathrm{TF}}(r)+V_{\text {core }}(r)+V_{\mathrm{LS}}(r)
$$

where $V_{\mathrm{TF}}(r)$ is the Thomas-Fermi potential, $V_{\text {core }}(r)$ is a core polarization potential, and the spin-orbit interaction is described by $V_{\mathrm{LS}}(r)$. The pseudopotential, $V_{\mathrm{He}}^{\mathrm{sph}}(r)$, describes the effective interaction of the valence electron and the $\mathrm{Cs}^{+}$core with the surrounding helium atoms. For a single helium atom, the latter interaction is given by

$V_{\mathrm{He}}(\mathbf{r}, \mathbf{R})=V_{\mathrm{e}-\mathrm{He}}(\mathbf{r}, \mathbf{R})+V_{\text {pol- } \mathrm{He}}(\mathbf{r}, \mathbf{R})+V_{\text {cross }}(\mathbf{r}, \mathbf{R})+V_{\text {cc }}(\mathbf{R})$

$V_{\mathrm{e}-\mathrm{He}}(\mathbf{r}, \mathbf{R})$ describes the Pauli repulsion of the valence electron by the closed electron shell of a single $\mathrm{He}$ atom located at position $\mathbf{R}, V_{\text {pol-He }}(\mathbf{r}, \mathbf{R})$ represents the polarization of the $\mathrm{He}$ atom by the valence electron, $V_{\text {cross }}(\mathbf{r}, \mathbf{R})$ accounts for the simultaneous polarization of the $\mathrm{He}$ atoms by $\mathrm{Cs}$ electron and core, and $V_{\mathrm{cc}}(\mathbf{R})$ describes the polarization of a He atom by the $\mathrm{Cs}^{+}$core. The parameters of these contributions are given explicitly in ref 13 and references therein.

The (central) pseudopotential is given by the volume integration of eq 23 weighted by the (spherically symmetric) He number density, $\rho^{\text {sph }}(\mathbf{R})$, introduced below, and by the integration over the valence electron's angular degrees of freedom, according to

$$
V_{\mathrm{He}}^{\mathrm{sph}}(r)=\iint \rho^{\mathrm{sph}}(\mathbf{R}) V_{\mathrm{He}}(\mathbf{r}, \mathbf{R}) \mathrm{d} \mathbf{R} \mathrm{d} \Omega_{\mathrm{e}}
$$

The Schrödinger equation is then solved numerically for the electron with the resulting effective electron-He pseudopotential, together with the free-atomic potential and the centrifugal term in eq 20.

The spherical bubble is described by an $R$-dependent $\mathrm{He}$ density, that is usually parametrized as according to

$$
\frac{\rho^{\mathrm{sph}}\left(R, R_{0}, \varepsilon\right)}{\rho_{0}}= \begin{cases}0 & R<R_{0} \\ 1-\left\{1+\varepsilon\left(R-R_{0}\right)\right\} e^{-\varepsilon\left(R-R_{0}\right)} & R \geq R_{0}\end{cases}
$$

where $\varepsilon^{-1}$ characterizes the width of its smoothly varying boundary.

The bubble is thus described by the bulk density, $\rho_{0}$, and by the two parameters, $R_{0}$ and $\varepsilon$, that can be obtained by numerically minimizing the total bubble energy, $E_{\text {tot }}^{\text {bub }}$. The latter is given by the sum of the atomic energy, $E_{\text {eig }}$ and bubble energy terms, $E_{\text {bub }}$, which comprise the hydrostatic energies (pressure-volume work and surface tension), a kinetic energy term, $E_{\text {kin }}$, from the delocalization at the bubble boundary, and an energy, $E_{\text {elast }}$, due to the 
elastic deformation of the matrix.

$$
\begin{gathered}
E_{\text {tot }}^{\text {bub }}=E_{\text {eig }}+E_{\text {bub }} \\
E_{\text {bub }}=\frac{4}{3} \pi R_{\mathrm{b}}^{3} p+4 \pi R_{\mathrm{b}}^{2} \sigma+E_{\text {kin }}+E_{\text {elast }}
\end{gathered}
$$

$p$ is the bulk helium pressure and $R_{\mathrm{b}}$ is defined as a center of mass of the bubble boundary. One can solve this minimization problem and derive the bubble parameters for a liquid $\mathrm{He}$ matrix.

At the solidification point, the pressure remains practically unchanged but the density increases. The results of the minimization procedure show that, without inclusion of elastic forces, one gets a new equilibrium state corresponding to a bubble of different size but approximately the same energy. This theoretical result is in contradiction with the experimental findings. We therefore concluded $^{13}$ that there must be a restoring force in the solid that acts against the force exerted by the Cs valence electron on the bubble interface. It tends to compress the bubble and thus further downshifts the energies. In ref 13 , we have written this restoring force as

$$
F_{\text {eq.el. }}=-F_{\mathrm{Cs}-\mathrm{He}}=-\left.\frac{\partial E_{\mathrm{eig}}}{\partial R_{0}}\right|_{R_{0}=R_{\mathrm{eq}}}
$$

where $R_{\mathrm{eq}}$ is the equilibrium bubble radius for the given electronic state $\left(6 S_{1 / 2}\right.$ or $\left.6 P_{1 / 2}\right)$. This correction to the SBM yields a better description of the Cs atom in solid He than the "liquid" model, which ignores elasticity. However, the elastic correction overestimates the $\mathrm{D}_{1}$ excitation line shift, whereas the calculated fluorescence line center falls in between the experimental values for bcc and hcp.

\section{APPENDIX B. GENERALIZED YOUNG'S MODULUS} FOR HCP AND BCC HELIUM CRYSTALS

To include matrix anisotropy effects on the dopant atom in the continuous medium formalism, one needs to relate the elastic forces (stress) in the matrix to the deformation induced in the matrix by the atomic inclusion (strain). Such a relation must be based on the stress-strain tensor for the medium and the Eshelby tensor for the given inclusion shape. Related problems have been (numerically) solved for particular cases, such as for a spherical cavity in a cubic medium, ${ }^{34}$ or for an ellipsoidal cavity in an orthotropic medium. ${ }^{35}$ We address the anisotropy of the elastic properties of solid in the frame of the stress-strain formalism

$$
\sigma_{i, j}=C_{i j k l} \varepsilon_{k l}
$$

where $\sigma_{i j}$ denotes the stress in the medium induced by the strain $\varepsilon_{k l}$, and $C_{i j k l}$ is the 4 th order stiffness tensor. The lattice structures of bcc and hcp crystals have the symmetries $O_{h}^{9}$ and $D_{6 h}^{4}$, respectively, and the corresponding stiffness tensors have only a few nonzero matrix elements. In engineering notation, the stiffness tensors are replaced by $6 \times 6$ matrices (see, for example refs 36 and 37):

$$
\sigma_{i}=C_{i j} \varepsilon_{j}
$$

where

$$
C_{i j}^{\mathrm{hcp}}=\left(\begin{array}{cccccc}
c_{11} & c_{12} & c_{13} & 0 & 0 & 0 \\
c_{12} & c_{11} & c_{13} & 0 & 0 & 0 \\
c_{13} & c_{13} & c_{33} & 0 & 0 & 0 \\
0 & 0 & 0 & c_{44} & 0 & 0 \\
0 & 0 & 0 & 0 & c_{44} & 0 \\
0 & 0 & 0 & 0 & 0 & \frac{c_{11}-c_{12}}{2}
\end{array}\right)
$$

and

$$
C_{i j}^{\mathrm{bcc}}=\left(\begin{array}{cccccc}
c_{11} & c_{12} & c_{12} & 0 & 0 & 0 \\
c_{12} & c_{11} & c_{12} & 0 & 0 & 0 \\
c_{12} & c_{12} & c_{11} & 0 & 0 & 0 \\
0 & 0 & 0 & c_{44} & 0 & 0 \\
0 & 0 & 0 & 0 & c_{44} & 0 \\
0 & 0 & 0 & 0 & 0 & c_{44}
\end{array}\right)
$$

Values for the helium crystal coefficients $c_{i j}$ were reported in refs 7 and 31. The stiffness tensor leads to the anisotropic compressibility

$$
\kappa(\hat{\mathbf{n}})=n_{i} n_{j} C_{i j k l}^{-1} n_{k} n_{l}
$$

which gives the relative matrix compression due to a force applied along $\hat{\mathbf{n}}$. Figure 11 shows the directional dependence of the compressibility in bcc and hcp crystals, respectively.

\section{AUTHOR INFORMATION}

\section{Corresponding Author}

*E-mail: victor.lebedev@unifr.ch.

\section{Notes}

The authors declare that they have no competing financial interests.

\section{ACKNOWLEDGMENT}

This work was supported by Grant No. 200020-129831 of the Schweizerischer Nationalfonds. We thank Paul Knowles for his careful proofreading of the manuscript.

\section{REFERENCES}

(1) Toennies, J. P.; Vilesov, A. F. Angew. Chem., Int. Ed. 2004, $43,2622-2648$.

(2) Lebedev, V.; Moroshkin, P.; Toennies, J. P.; Weis, A. J. Chem. Phys. 2010, 133, 154508

(3) Crepin-Gilbert, C.; Tramer, A. Int. Rev. Phys. Chem. 1999, 18,485 .

(4) Moroshkin, P.; Hofer, A.; Ulzega, S.; Weis, A. Low Temp. Phys. 2006, 32, 981.

(5) Moroshkin, P.; Hofer, A.; Weis, A. Phys. Rep. 2008, 469, 1.

(6) Jortner, J; Kestner, N. R.; Rice, S. A.; Cohen, M. H. J. Chem. Phys. 1965, 43, 2614-2625.

(7) Greywall, D. S. Phys. Rev. B 1976, 13, 1056.

(8) Crepeau, R. H.; Heybey, O.; Lee, D. M.; Strauss, S. A. Phys. Rev. A 1971, 3, 1162.

(9) Bauer, H.; Beau, M.; Friedl, B.; Marchand, C.; Miltner, K.; Reyher, H. J. Phys. Lett. A 1990, 146, 134.

(10) Kanorsky, S.; Weis, A.; Arndt, M.; Dziewior, R.; Hänsch, T. W. Z. Phys. B 1995, 98, 371

(11) Moroshkin, P.; Hofer, A.; Nettels, D.; Ulzega, S.; Weis, A. J. Chem. Phys. 2006, 124, 024511.

(12) Nettels, D.; Hofer, A.; Moroshkin, P.; Müller-Siebert, R.; Ulzega, S; Weis, A. Phys. Rev. Lett. 2005, 94, 063001.

(13) Hofer, A.; Moroshkin, P.; Ulzega, S.; Nettels, D.; MüllerSiebert, R.; Weis, A. Phys. Rev. A 2007, 76, 022502.

(14) Kanorsky, S. I.; Lang, S.; Lücke, S.; Ross, S. B.; Hänsch, T. W.; Weis, A. Phy. Rev. A 1996, 54, R1010.

(15) Lang, S; Kanorsky, S. I.; Arndt, M.; Ross, S. B.; Hänsch, T. W.; Weis, A Europhys. Lett. 1995, 30, 233-237.

(16) Kanorsky, S.; Lang, S.; Eichler, T.; Winkler, K.; Weis, A. Phys. Rev. Lett. 1998, 81, 401.

(17) Lang, S.; Arndt, M.; Hänsch, T. W.; Kanorsky, S.; Lücke, S.; Ross, S. B.; Weis, A. Low Temp. Phys. 1996, 22, 129. 
(18) Melich, M.; Dupont-Roc, J.; Jacquier, P. J. Low Temp. Phys. 2008, 150, 301.

(19) Takahashi, Y.; Sano, K.; Kinoshita, T.; Yabuzaki, T. Phys. Rev. Lett. 1993, 71, 1035.

(20) Kinoshita, T.; Fukuda, K.; Yabuzaki, T. Phys. Rev. B 1996, 54, 6600-6607.

(21) Moriwaki, Y.; Morita, N. Eur. Phys. J. D 2005, 33, 323-327.

(22) Moriwaki, Y.; Inui, K.; Kobayashi, K.; Matsushima, F.; Morita, N. J. Mol. Struct. 2006, 786, 112.

(23) Moriwaki, Y.; Morita, N. Eur. Phys. J. D 2001, 13, 11.

(24) Kinoshita, T; Takahashi, Y.; Yabuzaki, T. Phys. Rev. B 1994, 49, 3648.

(25) Furukawa, T.; Matsuo, Y.; Hatakeyama, A.; Fukuyama, Y.; Kobayashi, T.; Izumi, H.; Shimoda, T. Phys. Rev. Lett. 2006, 96, 095301.

(26) Arndt, M.; Kanorsky, S. I.; Weis, A.; Hänsch, T. W. Phys. Rev. Lett. 1995, 74, 1359.

(27) Arndt, M.; Dziewior, R.; Kanorsky, S.; Weis, A.; Hänsch, T. W. Z. Phys. B 1995, 98, 377.

(28) Kinoshita, T.; Fukuda, K.; Takahashi, Y.; Yabuzaki, T. Phys. Rev. A 1995, 52, 2707-2716.

(29) Edwards, D. O.; Pandorf, R. C. Phys. Rev. 1965, 140, 816.

(30) Edwards, D. O.; Pandorf, R. C. Phys. Rev. 1966, 144, 143.

(31) Greywall, D. S. Phys. Rev. B 1977, 16, 5127.

(32) Pascale, J. Phys. Rev. A 1983, 28, 632.

(33) Sobelman, Atomic Spectra and Radiative Transitions; Springer Series on Atoms and Plasmas; Springer: New York, 1992.
(34) Chiang, C.-R. J. Strain Anal. 2007, 42, 155.
(35) Kirilyuk, V.; Levchuk, O. Int. Appl. Mech. 2007, 43, 403.
(36) Paszkiewicz, T.; Wolski, S. Phys. Status Solidi B 2007, 244, 978.
(37) Paszkiewicz, T.; Wolski, S. Phys. Status Solidi B 2007, 244, 1. 